\title{
PREVALÊNCIA DE MEGAS EM NECRÓPSIAS REALIZADAS NO TRIÂNGULO MINEIRO NO PERÍODO DE 1954 A 1988
}

\section{Edison Reis Lopes, Ademir Rocha, Antônio Carlos O. Meneses, Marco Antonio B. Lopes, Márcio Cunha Fatureto, Gesner Pereira Lopes e Edmundo Chapadeiro}

\begin{abstract}
Dentre 1708 necrópsias de chagásicos crônicos, de um total de 4690, diagnosticamos 273 megas. Destes o mais freqüente foi o megacólon, seguido pelo megaesôfago, ocupando a associação megacólon e megaesôfago o terceiro lugar. Discutem-se e comparam-se os achados com outros de ordem clinico-epidemiológica e anatomopatológica. Confirmando dados da literatura, nossos achados atuais mostram, que a exemplo do que sucede na cardiopatia chagásica, o megacólon e o megaesôfago predominam no sexo masculino, discutindo-se os fatores que poderiam explicar a razão desse fato.
\end{abstract}

Palavras-chaves: Doença de Chagas. Megas. Megaesôfago. Megacólon.

Em algumas regiões do Brasil onde a Doença de Chagas é endêmica ocorre alta prevalência de megas do tubo digestivo. Trabalhos de ordem clínico-epidemiológical 2479111314151617252830313435 37384143 e anatomopatológica 35101921222324 demonstram não só a relação causa-efeito entre infecção chagásica e megas, como também vários deles preocupam-se com a prevalência e a distribuição destas visceromegalias quanto à sede, idade e sexo. Entretanto, a análise da mencionada literatura mostra divergências entre os resultados dos vários autores, mesmo quando se comparam dados de mesma natureza.

Tendo tido oportunidade de realizar no Triângulo Mineiro, 4690 autópsias, das quais cerca de um terço em chagásicos, pareceu-nos justificada uma nova análise da prevalência e distribuição dos megas neste material. Deve-se realçar que, ao que nos consta, nos dois últimos decênios não se realizaram levantamentos necroscópicos sobre o assunto e dos estudos efetuados até 1970 alguns basearam-se em casuísticas relativamente pequenas.

\section{MATERIAL E MÉTODOS}

Consta de 4690 necrópsias, realizadas no Departamento de Patologia, Medicina Legal e Deontologia Médica da Faculdade de Medicina do Triângulo

\footnotetext{
Trabalho dos Departamentos de Patologia e Medicina Legal da Faculdade de Medicina do Triângulo Mineiro e da Universidade Federal de Uberlândia.
}

Endereço para correspondência: Prof. Edison Reis Lopes, Faculdade de Medicina do Triângulo Mineiro, Praça Manoel Terra, s/n - 38025 Uberaba, MG, Brasil.

Recebido para publicação em 18/08/89.
Mineiro e do CEBIM da Universidade Federal de Uberlândia. Nestes serviços são realizadas, não só autópsias provenientes dos Hospitais-Escolas daquelas Instituições, como também dos Serviços de Verificação de Óbitos e dos Postos Médico-Legais. Para estes dois últimos são enviados os casos de morte. natural com causa mortis não esclarecida, bem como os de óbito violento (acidentes, homicídios, suicidios).

O diagnóstico de Doença de Chagas foi estabelecido com base nos achados sorológicos e/ou morfológicos 27 . O diagnóstico de mega foi baseado nos critérios propostos por Tafuri e Raso ${ }^{42}$ e por Lopes e cols $^{27}$.

As variáveis continuas foram avaliadas através do teste " $t$ " $e$ os dados e freqüência pelo "quiquadrado" sendo considerado significante quando o valor de "t" foi menor que $0,01(1 \%)$.

\section{RESULTADOS}

A Tabela 1 indica a distribuição dos vários tipos de megalias, a percentagem de ocorrência de cada tipo no total dos megas observados, bem como a freqüência relativa de cada visceromegalia entre os chagásicos e no total geral das necrópsias.

$\mathrm{Na}$ Tabela 2, distribuem-se os tipos mais freqüentes de megas: megacólon (MC), megaesôfago (ME) e associação megaesôfago-megacólon (ME + $\mathrm{MC)}$, segundo o sexo e a idade, à época do óbito.

A análise estatistica mostrou não haver diferença significativa, quanto à idade do óbito, entre os grupos $\mathrm{MC}, \mathrm{ME}$ e $\mathrm{MC}+\mathrm{ME}$.

\section{DISCUSSÃO}

Como indica a Tabela 1, dos 1708 chagásicos por nós necropsiados, 273 apresentaram um ou mais tipos de megas, o que equivale a uma freqüência de 
Lopes ER, Rocha A, Meneses ACO, Lopes MAB, Fatureto MC, Lopes GP, Chapadeiro E. Prevalência de megas em necrópsias realizadas no Triângulo Mineiro no periodo de 1954 a 1988. Revista da Sociedade Brasileira de Medicina Tropical 22: 211-215, Out-Dez, 1989.

Tabela 1 - Prevalência de megas em autópsias realizadas no Triângulo Mineiro no periodo 1954-1988.

\section{Freqüência relativa}

\begin{tabular}{lrccc} 
Tipos de mega & $\begin{array}{c}\text { N. } \\
\text { casos }\end{array}$ & $\begin{array}{c}\text { Megas } \\
(\mathrm{n}=273)\end{array}$ & $\begin{array}{c}\text { Chagásicos } \\
(\mathrm{n}=1708)\end{array}$ & $\begin{array}{c}\text { Necrópsias } \\
(\mathrm{n}=4690)\end{array}$ \\
\hline MC & 142 & 52,00 & 8,31 & 3,03 \\
ME & 71 & 26,00 & 4,16 & 1,51 \\
MC + ME & 48 & 17,50 & 2,81 & 1,02 \\
MC + ME + MVb & 2 & 0,70 & 0,12 & 0,04 \\
MC + MD & 2 & 0,70 & 0,12 & 0,04 \\
MC + ME + MEst & 2 & 0,70 & 0,12 & 0,04 \\
MC + MA & 1 & 0,40 & 0,06 & 0,02 \\
MC + ME + MD & 1 & 0,40 & 0,06 & 0,02 \\
MC + ME + MVb + MB & 1 & 0,40 & 0,06 & 0,02 \\
ME + MEst + MD & 1 & 0,40 & 0,06 & 0,02 \\
ME + MEst & 1 & 0,40 & 0,06 & 0,02 \\
ME + MD & 1 & 0,40 & 0,06 & 0,02 \\
\hline
\end{tabular}

\begin{tabular}{lllll}
\hline Total & 273 & 100,00 & 16,00 & 5,80
\end{tabular}

$\overline{\mathrm{MC}}=$ Megacólon; $\mathrm{ME}=$ Megaesôfago; MEst $=$ Megaestômago; $\mathrm{MVb}=$ Megavesicula biliar; $\mathrm{MD}=$ Megaduodeno; $\mathrm{MA}=$ Megaapêndice; $\mathrm{MB}=$ Megabexiga .

Tabela 2 - Distribuição dos casos de megacólon, megaesôfago e megacólon + megaesôfago segundo idade e sexo.

\begin{tabular}{|c|c|c|c|c|c|c|c|}
\hline \multirow{3}{*}{ Tipo de mega } & \multirow{2}{*}{\multicolumn{3}{|c|}{$\begin{array}{l}\text { Idade } \\
\text { (anos) }\end{array}$}} & \multicolumn{4}{|c|}{ Sexo } \\
\hline & & & & \multicolumn{2}{|c|}{ Masculino } & \multicolumn{2}{|c|}{ Feminino } \\
\hline & $\overline{\mathrm{X}} \pm \mathrm{S}$ & $\mathrm{Mx}$ & $\mathrm{Mn}$ & № casos & $\%$ & № casos & $\%$ \\
\hline Megacólon & $50,51 \pm 14,15$ & 82 & 19 & 77 & 54,2 & 65 & 45,8 \\
\hline Megaesôfago & $46,57 \pm 16,27$ & 82 & 27 & 58 & 81,7 & 13 & 18,3 \\
\hline $\begin{array}{l}\text { Megacólon + } \\
\text { Megaesôfago }\end{array}$ & $53,40 \pm 14,10$ & 78 & 17 & 38 & 79,2 & 10 & 20,8 \\
\hline
\end{tabular}

$\overline{\mathrm{X}}=$ Média de idade $\mathrm{S}=$ Desvio-padrão; $\mathrm{Mx}=$ Idade máxima; $\mathrm{Mn}=$ Idade minima.

$5,8 \%$ no total dos indivíduos por nós necropsiados e a $16 \%$ entre os chagásicos. Estes valores estão bem próximos daqueles observados por Barbosa e cols 5 , em Belo Horizonte, e por Chapadeiro e cols ${ }^{10}$, em Uberaba, que constataram, respectivamente, $16,6 \% \mathrm{e}$ $17,3 \%$ dos megas em pacientes com cardite chagásica crônica. Por outro lado, os valores de nossos dados atuais, bem como os de Barbosa e cols 5 e os de Chapadeiro e cols ${ }^{10}$, são bastante inferiores àqueles observados por Köberle 21, em Ribeirão Preto. Este último autor, em $1962^{21}$ e em $1968^{23}$, observou em seu material que $63,2 \%$ e $39,4 \%$, respectivamente, dos chagásicos por ele autopsiados, tinham megas. Aliás os quatro últimos trabalhos citados e o de Andrade e $\mathrm{col}^{3}$, em Salvador (que observaram megas em 2,37\% de suas necrópsias) são, ao que nos consta, os cinco únicos estudos anatomopatológicos sistematizados sobre o assunto. Várias razões podem ser invocadas para tentar explicar as diferenças entre os resultados do presente estudo e os anteriores realizados em Minas Gerais 5 10, em Ribeirão Preto 2123 e em Salvador ${ }^{3}$. Dentre estas razões ressaltam-se as 
Lopes ER, Rocha A, Meneses ACO, Lopes MAB, Fatureto MC, Lopes GP. Chapadeiro E. Prevalência de megas emnecropsias realizadas no Triângulo Mineiro no periodo de 1954 a 1988. Revista da Sociedade Brasileira de Medicina Tropical 22: 211-215, Out-Dez, 1989.

eventuais diferenças geográficas ${ }^{10} \mathrm{e}$ os criterios empregados para o diagnóstico morfológico, especialmente naqueles casos em que a dilatação da viscera e pouco significativa.

No que se refere à viscera acometida, os estudos anatomopatológicos prévios de Barbosa e cols 5 e Köberle 2123 e os nossos atuais indicam ser o megacólon (MC) o tipo mais freqüente de mega no chagásico. Em seguida situam-se o megaesôfago (ME) e a associação megacólon-megaesõfago (MC $+\mathrm{ME})$. Os dados de Chapadeiro e col em $1964^{10}$ mostraram que ME e MC ocupavam o primeiro lugar, seguidos pelo $\mathrm{MC}+\mathrm{ME}$ e o trabalho de Andrade e $\mathrm{col}^{1}{ }^{3}$ indicou em primeiro lugar, com igual prevalência, $\mathrm{MC}$ e $\mathrm{MC}+$ ME e em terceiro lugar ME. Deve-se lembrar, entretanto, que a casuística, nestes dois últimos estudos, foi muito pequena quando comparada a de Köberle ${ }^{21} 23$, Barbosa e $\operatorname{col}^{5}$ e a nossa atual.

Em oposição a estes dados morfológicos, trabalhos baseados em estudos clínicos e/ou radiológicos parecem indicar ser o $\mathrm{ME}$ a visceromegalia prevalente no chagásico ${ }^{1} 9283339$. Entretanto, Dias ${ }^{11} \mathrm{em}$ estudo de campo, em Bambui (MG), baseado na análise clínica e radiológica, observou nos chagásicos crôni$\cos 28,7 \%$ de colopatias para $18,3 \%$ de esofagopatias. Também Atias ${ }^{4}$, no Chile, baseado em estudos clínicos, afirma ser o MC bem mais freqüente que o $\mathrm{ME}$ e a associação $\mathrm{MC}+\mathrm{ME}(76 \%$ de $\mathrm{MC}$ para $17,7 \%$ de $\mathrm{ME}$ e $5,3 \%$ de $\mathrm{MC}+\mathrm{ME})$.

Hipóteses podem ser levantadas na tentativa de explicar também as divergèncias entre os achados anatomopatológicos e clínico-radiológicos. Primeiramente, porque o diagnóstico clínico de $\mathrm{ME}$ parece ser feito com mais frequência do que o de $\mathrm{MC}$, visto que à disfagia, principal manifestação do ME, é conferido, com freqüência, maior valor diagnóstico que a constipação, que costuma ser a queixa primordial no MC. Em segundo lugar, porque na maioria dos estudos clínicos e, especialmente, naqueles de campo, o exame radiológico é empregado para auxiliar no diagnóstico de ME, mas não é aplicado para o diagnóstico de MC. Em terceiro lugar, no diagnóstico clínico de $\mathrm{ME}$, com relativa freqüência se empregam critérios como os de Rezende e $\mathrm{col}^{40}$, para quem, no megasesfôfago grupo I, o órgão é de calibre aparentemente normal ao exame radiológico, trânsito lento e pequena retenção de contraste. Ora, o anatomopatologista não diagnostica megaesôfago em órgão com calibre normal. Deve-se relembrar que estudos nas áreas endèmicas de Mambai $^{8}$, Virgem da Lapa ${ }^{32}$ e Bambui ${ }^{12}$ mostraram que $63,1 \%, 67,9 \%$ e $72 \%$ dos portadores de ME estavam incluidos neste grupo $\mathrm{I}$.

Não incluimos em nosso estudo as bronquiectasias, que na estatística de Köberle 22 ocorreram em
30 dos 500 (6\%) chagásicos crônicos por ele necropsiados. A razão da exclusão deste tipo de lesão brônquica é baseada no fato de que, ao contrário do que parece suceder em outras visceromegalias, estudos de vários autores (ver revisão da literatura em Bethlem e cols ${ }^{6}$ ) não confirmam relação de causaefeito entre a tripanossomíase e bronquiectasia.

Outro dado interessante da nossa casuística é o da distribuição dos diferentes tipos de megas, entre os sexos. Dados epidemiológicos, obtidos em estudos de campo $^{9} 29$, Tavares Neto, J (dados não publicados) mostram não haver diferença significativa na prevalência da infecção chagásica entre homens e mulheres. Isto contrasta com o que se observa em relação à prevalência de cardiopatia chagásica, a qual é bem maior no homem que na mulher ${ }^{29}$. Nossos dados atuais e os de outros autores mostram qua o MC e c ME predominam também no sexo masculino. Este predomínio é nítido no $\mathrm{ME}$ e bem menos acentuado no MC. Dias ${ }^{11}$, em Bambuí, MG, observou que a colopatia foi significantemente maior no sexo feminino, enquanto que para a esofagopatia não houve diferença de prevalência quanto aos sexos. A que, eventualmente, se atribuir essa diferença entre os sexos na cardiopatia e nos megas? Com relação à cardiopatia, o predominio do sexo masculino tem sito atribuido por alguns autores (ver literatura em Lopes 26 ) à maior atividade física do homem em comparação com a mulher. Este raciocínio, obviamente, não pode ser aplicado aos megas digestivos. Os achados de Widmer e coll4, de Pugliese e $\mathrm{col}^{36}$ e de Hauschka ${ }^{18}$, mostrando, tanto no homem como em condições experimentais, predominio da parasitemia no sexo masculino, talvez pudessem contribuir para a explicação da diferença observada.

Finalmente, quanto à idade em que ocorre o óbito, não detectamos diferença significativa entre os grupos $\mathrm{MC}, \mathrm{ME}$ e $\mathrm{MC}+\mathrm{ME}$ e observamos que a morte atinge os pacientes predominantemente entre a 4a e 5a décadas. Quando se compara esta idade do óbito com aquela observada nos chagásicos cardiopatas, sem megas, constata-se que nestes últimos a morte é precoce. Dados por nós obtidos em trabalho prévio $^{26}$ mostraram que o cardiopata chagásico masculino morre subitamente com a idade média de 38,2 anos (desvio-padrão 8,82) e a mulher com 37,1 anos (desvio-padrão 11,01 ). Nos chagásicos com insuficiência cardíaca congestiva estes valores são 43,7 anos (desvio-padrão 12,94) e 41,4 anos (desvio-padrão $14,46)$ para o homem e mulher, respectivamente. Se compararmos estes valores dos cardiopatas sem megas com os nossos atuais dados nos chagásicos com megas, veremos que a diferença de idade é significativa, considerando-se nivel de significância menor que $1 \%(\mathrm{p}<0,01)$. 
Lopes ER, Rocha A, Meneses ACO, Lopes MAB, Fatureto MC, Lopes GP, Chapadeiro E. Prevalência de megas em necropsias realizadas no Triângulo Mineiro no periodo de 1954 a 1988. Revista da Sociedade Brasileira de Medicina Tropical 22: 211-215, Out-Dez, 1989.

\section{SUMMARY}

One thousand seven hundred and eight chronic chagasic post-mortem examinations studied from a total of 4690 autopsies perfomed at our Institution. Two hundred and seventy-three chagasic had megas. Megacolon was the most frequent, followed by megaesophagus. Megacolon associated with megaesophagus was the third most commom finding. Our data are discussed and compared with the literature. Megacolon and megaesophagus were more prevalent in man, as shown by other workers. Higher parasitemia perhaps could explain this finding.

Key-words: Chagas' disease. Megas. Megacolon. Megaesophagus.

\section{AGRADECIMENTOS}

Aos funcionários dos Departamentos de Patologia, Medicina Legal e Deontologia Médica da Faculdade de Medicina do Triângulo Mineiro e da Universidade Federal de Uberlândia pelo apoio técnico-administrativo. Aos professores Euclides Ayres Castilho e José Tavares-Neto pelo estudo estatístico.

\section{REFERÊNCIAS BIBLIOGRÁFICAS}

1. Alecrim WD, Castro CN, Rezende JM, Macedo V, Prata A.Estudo da dinâmica esofágica entre duas áreas endêmicas da Doença de Chagas. In: Resumos do XIII Congresso da Sociedade Brasileira de Medicina Tropical, Brasilia, p. 24, 1977.

2. Almeida Prado A. Mal de engasgo ou Doença de Chagas? (Bloqueio auriculo-ventricular). São Paulo Médico 1:95-112, 1945.

3. Andrade $Z$, Andrade SG. O coração nos megas do aparelho digestivo. O Hospital 71:719-726, 1967.

4. Atias A. Enfermedad de Chagas digestiva en Chile. Boletin Chileno de Parasitologia 24:70-74, 1969.

5. Barbosa AJA, Pittella JEH, Tafuri WL. Incidência de cardiopatia chagásica em 15.000 necrópsias consecutivas e sua associação com os megas. Revista da Sociedade Brasileira de Medicina Tropical 4:219-223, 1970.

6. Bethlem NM, Lemle A, Saad E. Pulmonary manifestations in Chagas' disease. Seminars in Respiratory Medicine 9:432-439, 1988.

7. Brasil A. Aperistalsis of the oesophagus. Revista Brasileira de Gastroenterologia 7:21-44, 1955.

8. Castro C, Rezende JM, Camargo M, Prata A, Macêdo V. Prevalência de esofagopatia chagásica no município de Mambai, Goiás, Brasil. Revista da Sociedade Brasileira de Medicina Tropical 20:13-17, 1987.

9. Castro $\mathrm{CN}$. Influência de parasitemia no quadro clinico da Doença de Chagas. Tese de mestrado, Universidade de Brasília, Brasilia, 1978.

10. Chapadeiro E, Lopes ER, Mesquita PM, Pereira FEL. Incidência de megas associados à cardıopatia chagásica. Revista do Instituto de Medicina Tropical de São Paulo 6:287-291, 1964
11. Dias JCP. Doença de Chagas em Bambui, Minas Gerais, Brasil. Estudo clínico-epidemiológico a partir da fase aguda entre 1940 e 1982 . Tese de doutorado, Faculdade de Medicina da Universidade Federal de Minas Gerais, Belo Horizonte, 1982.

12. Dias JCP, Camacho LAB, Silva JC, Magalhães JS, Krieger H. Esufagopatia chagásica na área endêmica de Bambui, MG, Brasil, Revista da Sociedade Brasileira de Medicina Tropical 16:46-57, 1983.

13. Doria OBS. Conduta na oclusão aguda do megacólon. Revista Brasileira de Gastroenterologia 5:379-392, 1953.

14. Faria CAF. Condições de saúde entre trabalhadores rurais no município de Luz, Minas Gerais, com especial atenção à prevalência e à morbidade da Doença de Chagas. Tese de doutorado, Faculdade de Medicina da Universidade Federal de Minas Gerais, Belo Horizonte, 1978.

15. Finocchiaro J. Megacolo total: contribuição para a etiopatogenia dos megas. Medicina Cirurgia Farmácia 269:377-388, 1958

16. Freitas JLP. Contribuiçāo para o estudo do diagnóstico da moléstia de Chagas por processo de laboratório. Tese. Faculdade de Medicina da Universidade de São Paulo, São Paulo, 1947.

17. Freitas Júnior SV. Megacolo e megaesôfago no Brasil Central. Revista Clínica Cientifica 19:411-423, 1950.

18. Hauschka TS. Sex of host as a factor in Chagas' disease. Journal Parasitology 33:399-404, 1947.

19. Köberle F. Patogênese dos megas. Revista Goiana de Medicina 2:101-110, 1956.

20. Köberle F. Patologia da moléstia de Chagas. Medicina Centro Acadêmico Rocha Lima 1:73-98, 1962.

21. Köberle F. Pathologic anatomy of enteromegaly in Chagas disease. In: Reis FA dos (ed) Proceedings, 2a edição, Imprensa da Universidade de Minas Gerais, Rio de Janeiro, p. 92-103, 1962.

22. Köberle F. Patogenia do megaesôfago brasileiro e europeu. Revista Goiana de Medicina 9:79-116, 1963.

23. Köberle F. Patogenia da moléstia de Chagas. In: Cançado JA (ed) Doença de Chagas, Imprensa Oficial do Estado de Minas Gerais, Belo Horizonte, p. 238-259, 1968.

24. Köberle F, Nador E. Etiologia e patogenia do megaesôfago no Brasil. Revista Paulista de Medicina 47:643$661,1955$.

25. Laranja FS, Dias E, Nóbrega G. Estudo eletrocardiográfico de 81 casos de megaesôfago. Primeiro Congresso Panamericano de Medicina, Rio de Janeiro, 1946.

26. Lopes ER. Morte súbita em área endèmica da Doença. de Chagas. Sua importância médico-legal. Tese de Professor Titular, Faculdade de Medicina do Triângulo Mineiro, Uberaba, 1981.

27. Lopes ER, Chapadeiro E, Tafuri WL, Prata AR Patologia das Principais Doenças Tropicais no Brasil. Doença de Chagas. In: Lopes ER, Chapadeiro E, Raso P, Tafuri WL (ed), Bogliolo Patologia, 4! edição, Guanabara, Rio de Janeiro, p. 1047-1120, 1987.

28. Macedo VO. Influência de exposição à reinfecção na evolução da Doença de Chagas (Estudo longitudinal de cinco anos). Tese de Livre-Docência, Faculdade de 
Lopes ER, Rocha A, Meneses ACO, Lopes MAB, Fatureto MC, Lopes GP, Chapadeiro E. Prevaléncia de megas em necropsias realizadas no Triângulo Mineiro no periodo de 1954 a 1988. Revista da Sociedade Brasileira de Medicina Tropical 22: 211-.15, Out-Dez, 1989.

Medicina da Universidade Federal do Rio de Janeiro, Rio de Janeiro, 1973.

29. Nogueira JL. Levantamento epidemiológico sobre c irdiopatias e pressão arterial na população do Municílio de Cássia dos Coqueiros, São Paulo, Brasil. Tese de Doutorado, Faculdade de Medicina da Universidade de São Paulo, Ribeirão Preto, 1972.

30. Nunan B, Rezende CL, Canelas A. Contribuição ao tema Doença de Chagas na infância. Anais da VI Jornada Brasileira de Puericultura e Pediatria, Belo Horizonte, p. 655-658, 1952.

31. Pellegrino J, Borrotchin M. Inquérito sobre Doença de Chagas no Hospital da Santa Casa de Misericórdia de Belo Horizonte (Minas Gerais, Brasil). Memórias do Instituto Oswaldo Cruz 46:419-457, 1948.

32. Pereira JB, Coura JR Morbidade da Doença de Chagas. Estudo seccional em uma área endêmica, Virgem da Lapa, Minas Gerais. Revista da Sociedade Brasileira de Medicina Tropical 19:139-148, 1986.

33. Pinotti HW. Aspectos sócio-econômicos do megaesôfago e megacolo. In: Raia AA (ed) Manifestações digestivas da Moléstia de Chagas, Sarvier, São Paulo, p. 22-24, 1983.

34. Porto C. Gastropatia chagásica crônica. Nota prévia. Revista Goiana de Medicina 1:43-54, 1955.

35. Prata A. Relação etiológica entre Doença de Chagas e megaesôfago. Anais do V Congresso Internacional sobre a Doença de Chagas, Rio de Janeiro, p. 1317-1336, 1963.

36. Pugliese C, Lessa I, Santos Filho A. Estudo da sobrevida na miocardite crônica de Chagas descompensada. Revis- ta do Instituto de Medicina Tropical de São Paulo 18:191-201, 1976.

37. Ramos A, Carneiro O. Estudo clínico, eletrocardiográfico e radiológico da cardiopatia chagásica crônica. Análise de 106 casos. Revista Goiana de Medicina 2:287-296, 1956.

38. Rezende JM. Megaesôfago por Doença de Chagas. Revista Goiana de Medicina 2:297-314, 1956.

39. Rezende JM. Manifestações digestivas da moléstica de Chagas. In: Cançado JR (ed) Doença de Chagas. Imprensa Oficial do Estado de Minas Gerais, Belo Horizonte, p. 442-480, 1968.

40. Rezende JM, Lauar KM, Oliveira AR. Aspectos clínicos e radiológicos da aperistalse do esôfago. Revista Brasileira de Gastroenterologia 12:247-262, 1960.

41. Rezende JM, Rassi A. Comprometimento do esôfago na moléstia de Chagas. Megaesôfago e cardiopatia. O Hospital 53:1-15, 1958.

42. Tafuri WL, Raso P. Anatomia patológica. In: Raia AA (ed) Manifestaçōes digestivas da Moléstia de Chagas, 1! edição, Sarvier, São Paulo, p. 61-79, 1983.

43. Villela E. A ocorrência da moléstia de Chagas nos hospitais de Belo Horizonte e na população de seus arredores. Anais da Faculdade de Medicina da Universidade de Minas Gerais 1:1-8, 1930.

44. Widmer CG, Azevedo ES. Sexo do hospedeiro humano e o desenvolvimento de formas parasitárias do Trypanosoma cruzi no miocárdio. Revista do Instituto de Medicina Tropical de São Paulo 14:109-113, 1972. 